\title{
The effectiveness of axillary reverse mapping in preventing breast cancer-related lymphedema: a meta-analysis based on randomized controlled trials
}

\author{
Xuhui Guo, Dechuang Jiao, Jiujun Zhu, Hui Xiao, Xin Zhao, Yue Yang, Yajie Zhao, Zhenzhen Liu \\ Department of Breast Disease, Henan Breast Cancer Center, The affiliated Cancer Hospital of Zhengzhou University \& Henan Cancer Hospital, \\ Zhengzhou, China \\ Contributions: (I) Conception and design: X Guo; (II) Administrative support: Z Liu; (III) Provision of study materials or patients: X Guo, D Jiao,; \\ (IV) Collection and assembly of data: X Guo, J Zhu, H Xiao, X Zhao; (V) Data analysis and interpretation: X Guo, X Zhao, Y Yang, Y Zhao; (VI) \\ Manuscript writing: All authors; (VII) Final approval of manuscript: All authors. \\ Correspondence to: Zhenzhen Liu. Department of Breast Disease, Henan Breast Cancer Center, The affiliated Cancer Hospital of Zhengzhou \\ University \& Henan Cancer Hospital, Zhengzhou 450008, China. Email: zlyyliuzhenzhen0800@zzu.edu.cn.
}

Backgroundk Here, we carried out an extensive meta-analysis to investigate the effectiveness of the use of axillary reverse mapping (ARM) during axillary lymph node dissection (ALND) in preventing breast cancerrelated lymphedema (BCRL).

Methods: Database searches to identify relevant randomized controlled trials (RCTs) were performed of MEDLINE (PubMed), Web of Science, Embase, and the Cochrane Library. Eligible articles with a publication date from database establishment to December 2020 were retrieved by combining keywords including: "breast cancer", "breast carcinoma", "breast neoplasm", “axillary reverse mapping”, "axillary lymph node dissection", "lymphatic arm drainage", and "lymphedema". Independent data extraction was conducted, and Review Manager (version 5.3) was used for statistical analyses.

Results: Five eligible RCTs were included in the meta-analysis. A total of 37 patients suffered arm lymphedema $(37 / 786,4.71 \%$ ) in the experimental group (ARM during ALND), compared with 164 arm lymphedemas $(164 / 873,18.79 \%)$ in the control group (ALND alone). The results showed that ARM during ALND was superior to ALND alone in reducing the incidence of BCRL [OR $=0.20,95 \%$ confidence intervals (CI): 0.13-0.29, $\mathrm{P}<0.00001]$; however, the 2 procedures did not differ significantly in terms of oncological safety or shoulder movement $(\mathrm{OR}=0.30,95 \% \mathrm{CI}: 0.03-2.96, \mathrm{P}=0.30$; OR $=0.44,95 \%$ CI: $0.14-$ $1.40, \mathrm{P}=0.17)$.

Conclusions: ARM during ALND can prevent and reduce the occurrence of BCRL in patients with earlystage BC during long-term follow-up. Due to the limited number of RCTs available, more in-depth, highquality RCTs are urgently needed to provide a reliable and convincing basis for the application of ARM during ALND.

Keywords: Axillary reverse mapping (ARM); breast cancer (BC); lymphedema; meta-analysis; axillary lymph node dissection (ALND)

Submitted Feb 17, 2021. Accepted for publication Apr 09, 2021.

doi: $10.21037 /$ gs-21-186

View this article at: http://dx.doi.org/10.21037/gs-21-186 


\section{Introduction}

Breast cancer (BC) affects more women than any other malignant tumor. In recent years, its incidence has increased at an annual rate of $1.4 \%$ to $3.9 \%$, but the overall mortality rate has been declining year by year (1). With the decline in the mortality rate, ensuring a good quality of life for the increasing number of patients with $\mathrm{BC}$ who are attaining long-term survival is vital. BC-related lymphedema (BCRL), a frequently occurring sequela of surgical and radiation treatment for BC, can seriously affect the quality of life of BC survivors (2). Of patients who receive axillary lymph node dissection (ALND), between $7 \%$ and $77 \%$ have upper-limb lymphedema (3). Sentinel lymph node biopsy (SLNB) cannot completely eliminate lymphedema. Studies have shown that the incidence of lymphedema in patients who undergo SLNB alone is $3 \%$ to $13 \%$ (4). The advent of SLNB means that unnecessary ALND can be avoided and the occurrence of lymphatic edema in the upper extremities is minimized. However, the risk of upper limb lymphedema after SLNB surgery is still as high as 7\% (5); therefore, SLNB cannot solved the problem of upper limb lymphedema entirely. Currently, ALND remains the standard surgical procedure recommended by the guidelines for BC patients with clinically node-positive axilla $(\mathrm{CN}+)$.

Axillary reverse mapping (ARM) technology was first proposed in 2007. This technique visualizes the upper limb lymphatic tube channels by means of blue dyes, fluorescence, or radioisotopes, which distinguish the lymphatic tubes of the arms from those of the breast, allowing them to be preserved during axillary surgery, thereby minimizing the risk of upper limb lymphedema (6). However, previous studies have not established uniform criteria for successfully identifying arm lymph nodes and the occurrence of postoperative lymphedema (7-9). Also, whether metastasis has occurred in the arm lymph nodes presents an important oncological safety issue for the application of ARM. Theoretically, lymphatic drainage is nearly always performed exclusively in either the breast or the arm: it is incredibly rare for arm nodes to be involved with BC. However, arm lymphatic drainage does serve a purpose in the prevention of arm and hand lymphedemas, and it does not adversely affect disease outcomes $(10,11)$. Studies have shown that ARM can ensure oncologic safety while reducing the risk of BCRL during SLND or ALND (7). One recent randomized controlled trial (RCT) indicated that in early BC, ARM aided in reducing the lymphedema rate and did not compromise oncologic safety (12). However, studies have also suggested that the upper limb lymphatic tube drainage system is not entirely different from the breast lymphatic tube drainage system, so retaining any branch of the arm lymph nodes may introduce a risk of metastasis (13-16).

Currently, there is only limited evidence available as to whether ARM is clinically significant in preventing BCRL due to scant patient data in reported studies. Moreover, established recommendations or guidelines have yet to be agreed. To further scrutinize the available data, we performed an up-to-date meta-analysis to examine the effectiveness of ARM for preventing BCRL by searching electronic databases for relevant RCTs of ARM during ALND and assessing the feasibility and oncological safety. We present the following article in accordance with the PRISMA reporting checklist (available at http://dx.doi. org/10.21037/gs-21-186).

\section{Methods}

\section{Search strategy}

This systematic review and meta-analysis was conducted in adherence to the Preferred Reporting Items for Systematic Reviews and Meta-analyses (PRISMA) guidelines (17). Computerized database searches were conducted of MEDLINE (PubMed), Web of Science, Embase, and the Cochrane Library. We retrieved RCTs of ARM during ALND with a publication date from database initiation to December 2020 by combining keywords including: "breast cancer", "breast carcinoma", "breast neoplasm", "axillary reverse mapping", "lymphatic arm drainage", and "lymphedema". Only studies with human participants were considered. Retrieved articles were subjected to title and abstract review, and the full texts of studies of potential relevance were accessed for evaluation in detail. Only studies meeting the eligibility criteria set out below were included in the meta-analysis.

\section{Inclusion criteria}

Two coauthors independently reviewed publications that met the following criteria: (I) study included female outpatients aged over 18 years presenting with a clinical histological diagnosis of BC (invasive ductal carcinoma, ductal carcinoma, lobular carcinoma, and intraductal carcinoma) who received ARM during ALND; and (II) 
study was an RCT with the full text published in English.

The exclusion criteria were as follows: (I) study participants had received previous axillary surgery, with the exception of needle biopsy or concurrent SLNB; (II) study participants had been monitored for lymphedema for fewer than 3 months, (III) study participants had a history of lymphedema in at least 1 arm, recurrent BC following conservative surgical treatment, or bilateral tumors; (IV) study participants included pregnant women; (V) case reports, abstracts, conference reports, reviews, or reports of other experiments.

\section{Data extraction and quality assessment}

For the meta-analysis, study characteristic information was extracted from each eligible article including: author; country; study design details; sample size; body mass index (BMI) and age of the participants; lymph node status; pathological type; identification rate of ARM nodes or lymphatics; metastatic rate of ARM nodes; lymphedema assessment methods; duration of follow-up; and other signs of lymphedema. If a disagreement arose, it was settled through discussion between the 2 reviewers or with a $3^{\text {rd }}$ party. Review Manager (version 5.3; The Cochrane Collaboration, Oxford, UK) was used to assess the quality of trials that followed a control-and-treatment-group design.

\section{Risk of bias}

Risk-of-bias evaluation for the RCTs was performed using the Cochrane Collaboration's tool for assessing risk of bias. The 5 eligible studies were evaluated for bias in the following areas: random sequence generation, allocation concealment, blinding, incomplete outcome data, selective outcome reporting, and other risks. For each item, studies were categorized as having "low risk", "unclear risk", or "high risk".

\section{Outcome indices of literature}

The primary outcome was the incidence of arm lymphedema. Oncological safety and other related symptoms, such as shoulder movement restriction, were considered to be secondary outcomes.

\section{Statistical analysis}

Data were extracted independently and data analysis was performed using RevMan statistical software version 5.3. (Cochrane Collaboration, http://tech.cochrane.org/ revman/download). Risk ratios (RRs) and $95 \%$ confidence intervals (CI) were computed for extracted data which had a dichotomous outcome. As described above, the risk-of-bias assessment was performed using the bias risk tool provided by the Cochrane Collaboration (18). The heterogeneity of eligible studies was tested using the Cochran Q test and $\mathrm{I}^{2}$ statistic. $\mathrm{I}^{2}$ statistics of $25 \%, 50 \%, 75 \%$ were stands for mild, medium and severe heterogeneity, respectively (19). A $P$ value of 0.05 was considered statistically significant. If heterogeneity was moderate to high $\left(\mathrm{P}<0.05, \mathrm{I}^{2} \geq 50 \%\right)$, the random-effects model was employed to calculate the $95 \%$ CI; otherwise, the fixed-effects model was adopted $(\mathrm{P}>0.05$, $\mathrm{I}^{2} \leq 50 \%$ ) (20). Moreover, sensitivity analysis was performed to assess the potential heterogeneity of the results.

\section{Results}

\section{Study selection}

The initial database searches retrieved 440 studies including 93, 170, 21, and 156 records on PubMed, Embase, the Cochrane Library, and Web of Science, respectively. After removing 374 duplicate articles, we reviewed 66 publications by their titles and abstracts, and identified 11 potentially suitable articles for further analysis. Of these 11 articles, 6 were found to be ineligible and were subsequently excluded. Finally, 5 RCTs which met the eligibility criteria were included in this meta-analysis. Figure 1 shows the study selection process.

\section{Characteristics of the included studies}

Table 1 displays the characteristic information of each eligible study. The 5 included RCTs involved 1,659 participants in total $(2,12,21-23)$. Across these studies, a total of 786 patients received ARM of the nodes and lymphatics during ALND and 873 patients received conventional ALND. Patients in the 5 RCTs were followed up for between 6 and 37 months. All participants in the RCTs had a clinically and histologically confirmed diagnosis of breast carcinoma (invasive ductal carcinoma, lobular carcinoma, intraductal carcinoma, or mixed). Among the included studies, there were 2 studies from China (22,23), 2 from Egypt (12,21), and 1 from the Netherlands (2). All of the included studies detailed the surgical procedures used to perform ARM. Moreover, in 


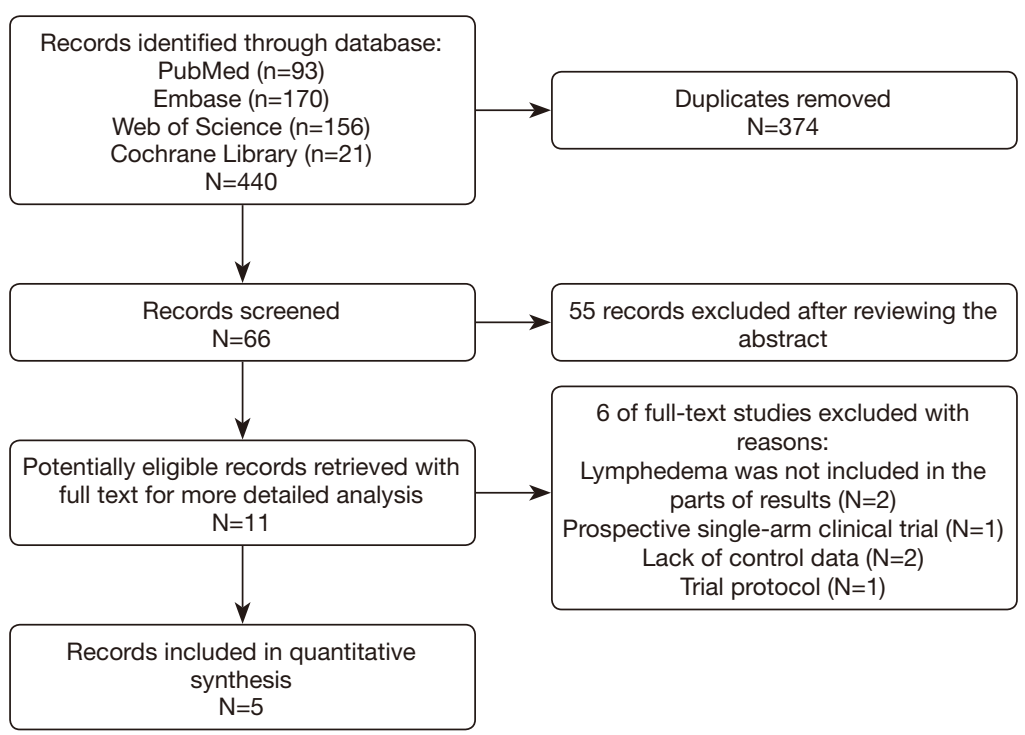

Figure 1 Flow diagram showing the process for selecting clinical trials for the meta-analysis.

one RCT, a novel, refined ARM technique was created, iDEntification and Preservation of ARm lymphaTic system (DEPART), which was then compared in combination with ALND against ALND alone, with the aim of examining the rates of arm lymphedema and recurrent locoregional disease (22). As for ARM mapping materials, 3 studies used blue dye alone $(2,12,21), 1$ study combined blue dye and fluorescence (22), and 1 study combined blue dye with radioisotope (23). With respect to the assessment of lymphedema, volumetric measurement was adopted in 3 studies, while the other 2 studies used arm circumference measurement. In the 5 included RCTs, the participants had clinical stage II or III. There were some differences between the 5 studies in terms of the included population. Patients presenting clinically with node-negative, early invasive $\mathrm{BC}$ were included in the studies by Abdelhamid et al. and Beek et al. $(2,12)$. However, in Yuan et al.'s 2019 study (22), patients with clinically diagnosed node-positive $\mathrm{BC}$ including patients who undergoing mastectomy for SLN-positive BC and breast-conserving surgery with at least 2 positive SLNs were the participants. Other studies had no specific description of the state of participants' armpit lymph nodes. All of the included studies evaluated the occurrence of lymphedema during different lengths of follow-up as well as oncological safety and treatmentassociated complications. Following the extraction of all relevant data, we performed the meta-analysis. Table 1 shows the study characteristic information.
The 5 included RCTs had a low to moderate risk of bias, as assessed using the Cochrane Collaboration's tool. The included studies showed a high quality (Figure S1). Three out of the 5 studies were multicenter RCTs $(2,21,22)$. Of the 5 studies, 3 used random number tables or computergenerated random numbers for random sequence generation, and 2 articles did not detail randomization methods. Three studies $(2,12,21)$ were participant- and assessor-blinded RCTs, whereas the remaining 2 studies did not mention blinding of participants or assessors $(22,23)$. One study (2) adopted adequate methods of allocation concealment and detailed how allocations were concealed. However, none of the other trials (12,21-23) described a concrete method for concealing allocations, which might have resulted in an elevated risk of bias for patient selection and measurement. For some of the studies the handling of missing data and the completeness of data collection were also unsatisfactory, resulting in a high risk of selection bias, which may have impacted the study outcomes.

\section{Primary outcomes: incidence of arm lymphedema}

All of the included studies reported the incidence of arm lymphedema as the primary outcome. Study participants were followed up for between 6 and 37 months. Across the aforementioned trials, a total of 37 patients suffered arm lymphedema $(37 / 786,4.71 \%)$ in the experimental 


\begin{tabular}{|c|c|c|c|c|c|c|c|c|c|c|c|c|c|}
\hline Author & Country & Sample $[\mathrm{N}]$ & Age & Condition of Iymph node & Pathological type & BMI & $\begin{array}{l}\text { Mapping } \\
\text { Material }\end{array}$ & Surgery & $\begin{array}{l}\text { Identification Rate } \\
\text { of ARM nodes or } \\
\text { lymphatics }\end{array}$ & $\begin{array}{l}\text { Metastatic } \\
\text { rate of ARM } \\
\text { nodes }\end{array}$ & $\begin{array}{l}\text { Assessment of } \\
\text { lymphedema }\end{array}$ & $\begin{array}{l}\text { Follow-up period } \\
\text { (months) }\end{array}$ & $\begin{array}{l}\text { Reported signs of } \\
\text { lymphedema }\end{array}$ \\
\hline \multirow[t]{2}{*}{$\begin{array}{l}\text { Abdelhamid } \\
\text { et al. (2020) }\end{array}$} & \multirow[t]{2}{*}{ Egypt } & ALND [49] & $56.5 \pm 7.5$ & \multirow[t]{2}{*}{$\begin{array}{l}\text { Clinically negative nodal metastasis } \\
\text { after positive SLNB, and were } \\
\text { admitted for completion ALND }\end{array}$} & $\begin{array}{l}\text { Ductal carcinoma } 44 \\
(89.8 \%) ; \text { Lobular carcinoma } \\
5(10.2 \%)\end{array}$ & $25.8 \pm 3$ & \multirow[t]{2}{*}{ Blue dye } & \multirow[t]{2}{*}{ NR } & \multirow[t]{2}{*}{ ALND (89/98) } & $1(2.3 \%)$ & \multirow[t]{2}{*}{$\begin{array}{l}\text { Volumetric } \\
\text { measurement }\end{array}$} & $32.6 \pm 7.2$ & \multirow[t]{2}{*}{$\begin{array}{l}\text { Shoulder movement } \\
\text { restriction }\end{array}$} \\
\hline & & ALND + ARM [49] & $57.4 \pm 6.5$ & & $\begin{array}{l}\text { Ductal carcinoma } 42 \\
\quad(85.7 \%) \\
\text { Lobular carcinoma } 7 \\
(14.3 \%)\end{array}$ & $26.3 \pm 3.3$ & & & & $0(0 \%)$ & & $33 \pm 7.1$ & \\
\hline Beek et al. (2019) & Netherlands & ALND [46] & 57.0 (mean) & $\begin{array}{l}\text { Clinicallly node negative, } \\
\text { scheduled for completion ALND } \\
\text { following a positive SLNB }\end{array}$ & early invasive breast cancer & 26.4 (mean) & Blue dye & NR & ALND (73/94) & $1 / 35$ & Volumetric measurement & 24 months & $\begin{array}{l}\text { Pain, paranesthesia, } \\
\text { numbness, loss of shoulder } \\
\text { mobility and the need to wear } \\
\text { a compression stocking }\end{array}$ \\
\hline \multirow{2}{*}{ Faisal et al. (2019) } & \multirow{2}{*}{ Egypt } & ALND [24] & $52 \pm 11$ & \multirow{2}{*}{$\begin{array}{l}\text { 29.3\% of participants had pNo } \\
\text { disease, 35.4\% had pN1 disease, } \\
\text { 18.8\% had pN2 disease, and } \\
16.7 \% \text { had pN3 disease }\end{array}$} & \multirow{2}{*}{ invasive ductal carcinoma } & \multirow{2}{*}{ NR } & \multirow{2}{*}{ Blue dye } & \multirow{2}{*}{$\begin{array}{l}\text { Conservative } \\
\text { breast surgery; } \\
\text { modified radical } \\
\text { mastectomy }\end{array}$} & \multirow{2}{*}{ ALND (20/24) } & \multirow{2}{*}{$0 / 4$} & \multirow{2}{*}{$\begin{array}{l}\text { Arm circumference } \\
\text { measurement }\end{array}$} & \multirow{2}{*}{6 months } & \multirow{2}{*}{ NR } \\
\hline & & ALND + ARM [24] & & & & & & & & & & & \\
\hline \multirow[t]{2}{*}{ Yuan et al. (2019) } & \multirow[t]{2}{*}{ China } & ALND [665] & 52.6 (mean) & \multirow[t]{2}{*}{ Clinically node-positive } & \multirow[t]{2}{*}{ Ductal; Lobular; Mixed } & 23.6 (mean) & \multirow{2}{*}{$\begin{array}{l}\text { Blue dye + } \\
\text { fluorescence }\end{array}$} & \multirow{2}{*}{$\begin{array}{l}\text { Mastectomy; } \\
\text { Breast- } \\
\text { conserving } \\
\text { surgery }\end{array}$} & \multirow[t]{2}{*}{ ALND (558/689) } & $38 / 558$ & Volumetric measurement & 37 months & NR \\
\hline & & ALND + ARM [689] & 51.3 (mean) & & & 23.4 (mean) & & & & & $\begin{array}{l}\text { and reported } \\
\text { subjectively by patients }\end{array}$ & & \\
\hline Yue et al. & China & ALND [127] & $50.52 \pm 9.95$ & Pathology node-positive & Invasive ductal & NR & Blue dye + & Modified radical & ALND (129/138) & $11 / 129$ & Arm circumference & 20 months & NR \\
\hline (2015) & & ALND + ARM [138] & $49.76 \pm 10.67$ & & $\begin{array}{l}\text { Carcinoma; Intraductal } \\
\text { carcinoma }\end{array}$ & & radioisotope & Mastectomy & & & measurement & & \\
\hline
\end{tabular}

ALND, axillary lymph node dissection; ARM, axillary reverse mapping. 


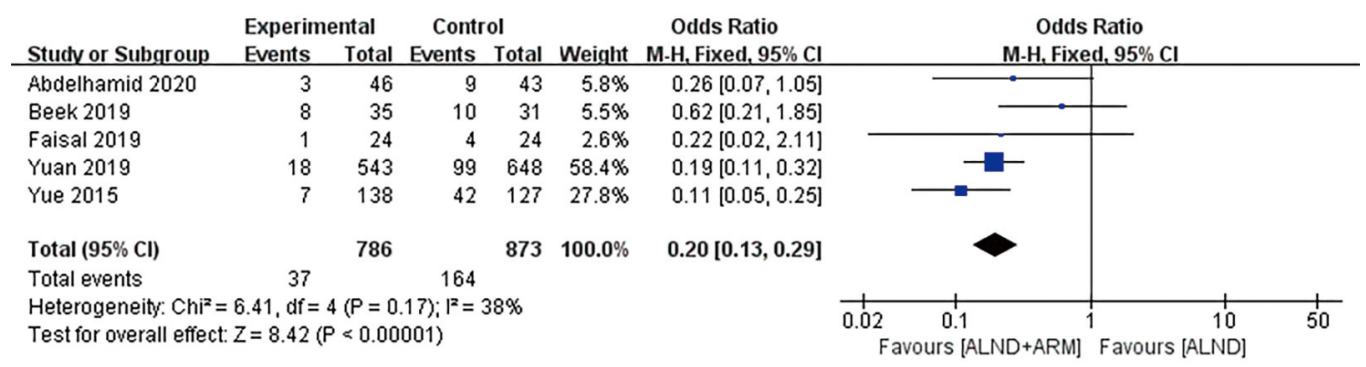

Figure 2 Forest plot of the included studies for the incidence of breast cancer-related lymphedema.

\begin{tabular}{|c|c|c|c|c|c|}
\hline \multirow[b]{2}{*}{ Study or Subgroup } & \multicolumn{2}{|c|}{ ALND+ARM } & \multicolumn{2}{|c|}{ ALND } & \multirow[b]{2}{*}{ Weight } \\
\hline & Events & Total & Events & Total & \\
\hline Abdelhamid 2020 & 0 & 46 & 1 & 43 & $49.9 \%$ \\
\hline Beek 2019 & 0 & 38 & 1 & 35 & $50.1 \%$ \\
\hline Total $(95 \% \mathrm{Cl})$ & & 84 & & 78 & $100.0 \%$ \\
\hline Total events & 0 & & 2 & & \\
\hline
\end{tabular}

Figure 3 Forest plot of the included studies for oncological safety.

group (ARM during ALND), while 164 arm lymphedemas $(164 / 873,18.79 \%)$ occurred in the control group (ALND alone). The RCTs displayed moderate heterogeneity $\left(\mathrm{P}=0.17, \mathrm{I}^{2}=38 \%\right)$; consequently, the fixed-effects approach was chosen. The results of pooled analysis revealed that the 2 groups differed significantly with respect to the incidence of arm lymphedema (OR $=0.20,95 \%$ CI: 0.13-0.29, $\mathrm{P}<0.00001)$ (Figure 2).

\section{Secondary outcomes: oncological safety and shoulder movement}

Oncological safety was reported in 2 studies, between which low-level heterogeneity was observed $\left(\mathrm{P}=0.99, \mathrm{I}^{2}\right.$ $=0 \%$; subsequently, the fixed-effects model was adopted. The results of pooled analysis failed to reveal a significant difference between the experimental and control groups in terms of oncological safety $(\mathrm{OR}=0.30,95 \% \mathrm{CI}$ : $0.03-2.96, \mathrm{P}=0.30)$. The same two studies also reported on shoulder movement, and significant heterogeneity was found between them $\left(\mathrm{P}=0.05, \mathrm{I}^{2}=73 \%\right)$; consequently, we chose the random-effects model. The results of pooled analysis showed that the experimental group and the control group did not differ significantly in terms of shoulder movement (OR $=0.44,95 \%$ CI: 0.14-1.4, $\mathrm{P}=0.17$ ) (Figures 3 and 4).

\section{Subgroup analyses}

Subgroup analyses were performed according to the followup time $(6,12$, or more than 20 months), study design (multicenter or single center), mapping material (blue dye, blue dye plus fluorescence, or blue dye plus radioisotope), and measurement of lymphedema (measurement of arm circumference or volume; subjective or objective measurement) (Figures 5-9).

Three studies reported data on the incidence of lymphedema over a 6-month follow-up period. Moderatelevel heterogeneity was found among these RCTs $(\mathrm{P}=0.24$, $\mathrm{I}^{2}=24 \%$ ); consequently, the fixed-effects model was adopted for data pooling. The overall estimate showed the pooled OR to be 0.70 (95\% CI: $0.32-1.51, \mathrm{P}=0.36$ ). The results of the subgroup analysis showed that the 2 groups did not differ significantly in terms of the incidence of BCRL during 6 months of follow-up. However, the results of the subgroup analysis for 12 and more than 20 months of follow-up differed from those for 6 months of followup. Moderate heterogeneity was observed among the experimental group and the control group $\left(\mathrm{P}=0.16, \mathrm{I}^{2}=46 \%\right.$ and $\mathrm{P}=0.15, \mathrm{I}^{2}=47 \%$, respectively); consequently, the fixedeffects model was chosen. The incidence of BCRL differed significantly between experimental group and control group $(\mathrm{OR}=0.18,95 \%$ CI: $0.10-0.33, \mathrm{P}<0.00001 ; \mathrm{OR}=0.23,95 \%$ CI: $0.15-0.36, \mathrm{P}<0.00001)$. 


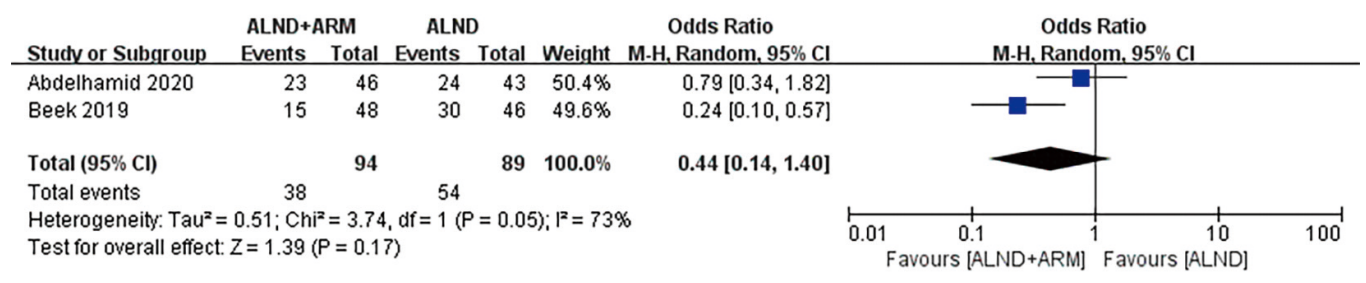

Figure 4 Forest plot of the included studies for shoulder movement.

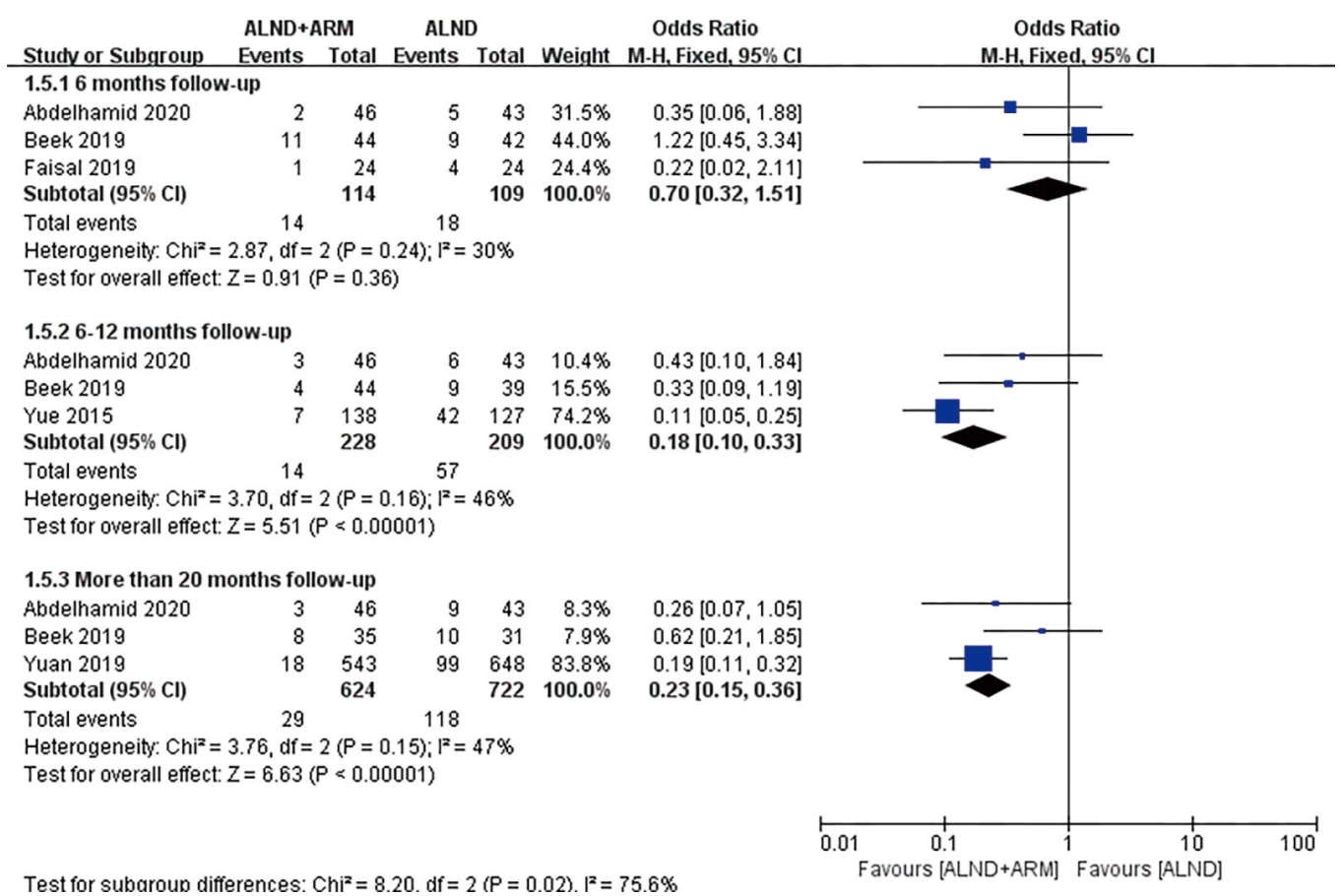

Figure 5 Forest plot for the incidence of breast cancer-related lymphedema in studies with 6, 12, and more than 20 months of follow-up.

The enrolled studies were also subjected to subgroup analysis depending on whether their study design was multicenter or single center. Three and 2 RCTs were conducted at multiple centers and a single center, respectively. The experimental group and the control group were revealed to be significantly different in reducing the incidence of BCRL $(\mathrm{OR}=0.23,95 \%$ CI: 0.14-0.36, $\mathrm{P}<0.00001 ; \mathrm{OR}=0.14,95 \%$ CI: $0.07-0.28, \mathrm{P}<0.00001)$.

Studies with data available on the mapping material used (blue dye, blue dye plus fluorescence, or blue dye plus radioisotope) were analyzed in subgroups. Three studies used blue dye as the mapping material, and the experimental group and the control group were found to different significantly ( $\mathrm{OR}=0.4,95 \%$ CI: $0.18-0.87, \mathrm{P}=0.02)$.

Two methods for the measurement of arm lymphedema were reported in the included studies: arm circumference measurement and volumetric measurement. A subgroup analysis was done to establish whether different measurement methods had an effect on the results. Pooled analysis using the fixed-effects model revealed the incidence of BCRL to differ between the experimental group and the control group of patients in both subgroups $(\mathrm{OR}=0.23,95 \%$ CI: $0.15-0.36, \mathrm{P}<0.00001 ; \mathrm{OR}=0.12$, 95\% CI: $0.05-0.26, \mathrm{P}<0.00001)$. Three studies reported the incidence rate of lymphedema using either subjective or objective measurement $(2,21,22)$. Pooled analysis under the random-effects model did not reveal significantly different incidences of BCRL in the subjective measurement subgroup (OR $=0.48,95 \% \mathrm{CI}: 0.13-1.85$, $\mathrm{P}=0.29)$. 


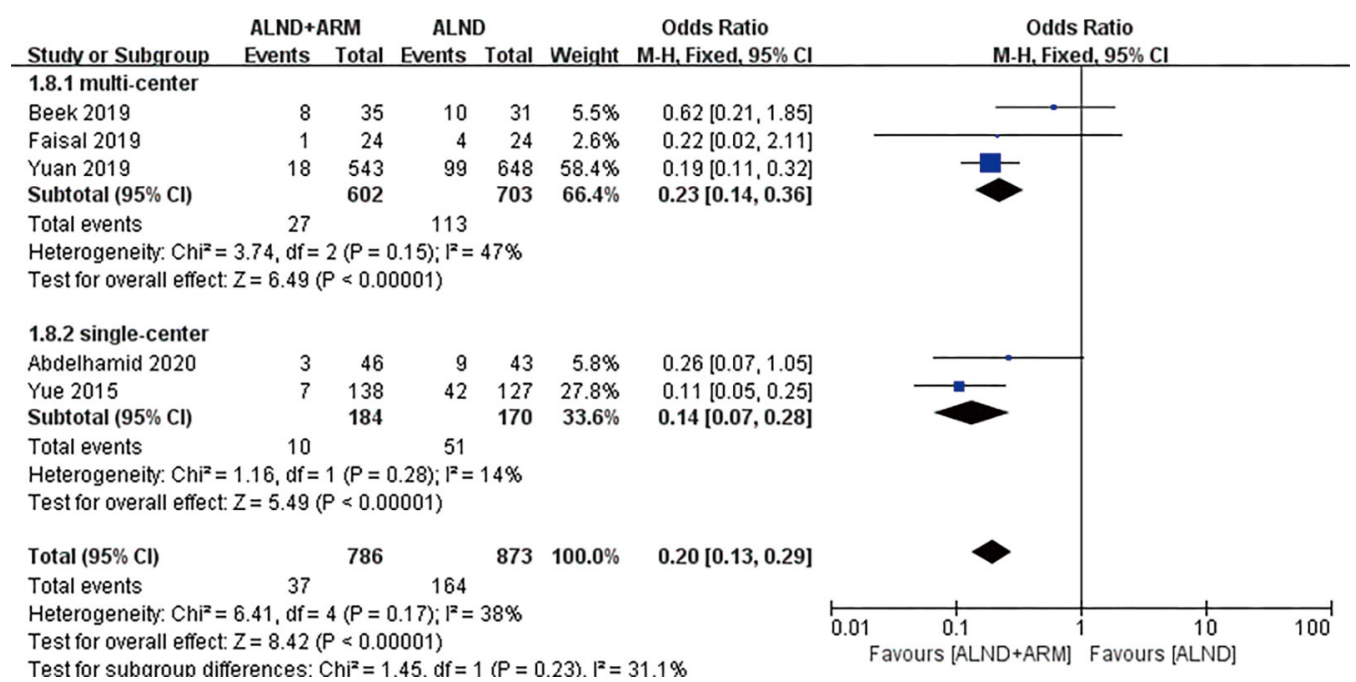

Figure 6 Forest plot for the incidence of breast cancer-related lymphedema in studies with different study designs (multicenter or single center).

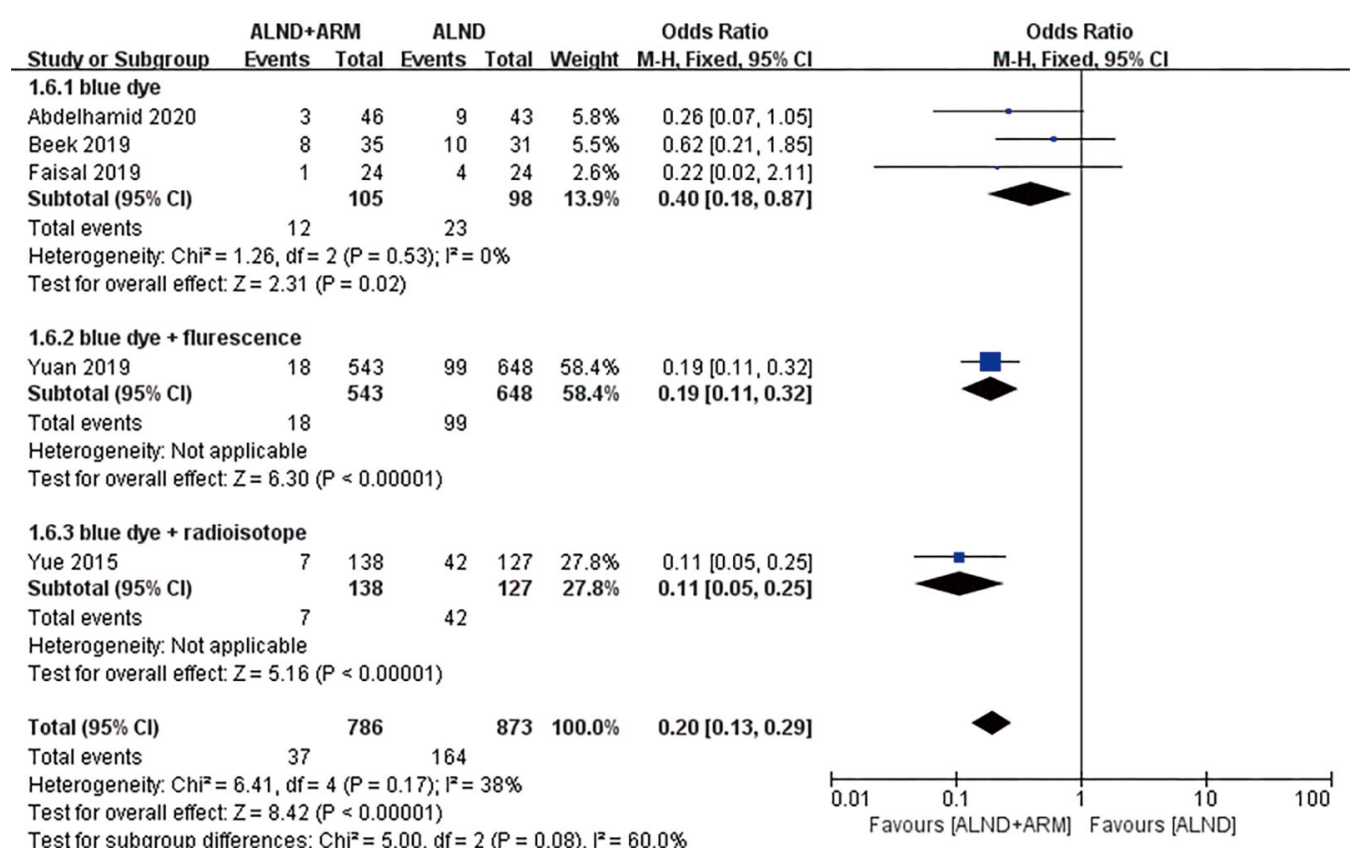

Figure 7 Forest plot for the incidence of breast cancer-related lymphedema in studies using different mapping materials.

\section{Sensitivity analysis}

Although the included articles were all RCTs, there are still heterogeneity existed in the meta-analysis. Therefore, we specifically conducted a sensitivity analysis to explore the source of heterogeneity. Each study was excluded in turn and the impact on the effect estimate was calculated. The effect estimate was not found to be affected by the exclusion of any one of the studies.

\section{Discussion}

The pathophysiological cause of BCRL is generally accepted to be axillary lymphatic blockage due to the 


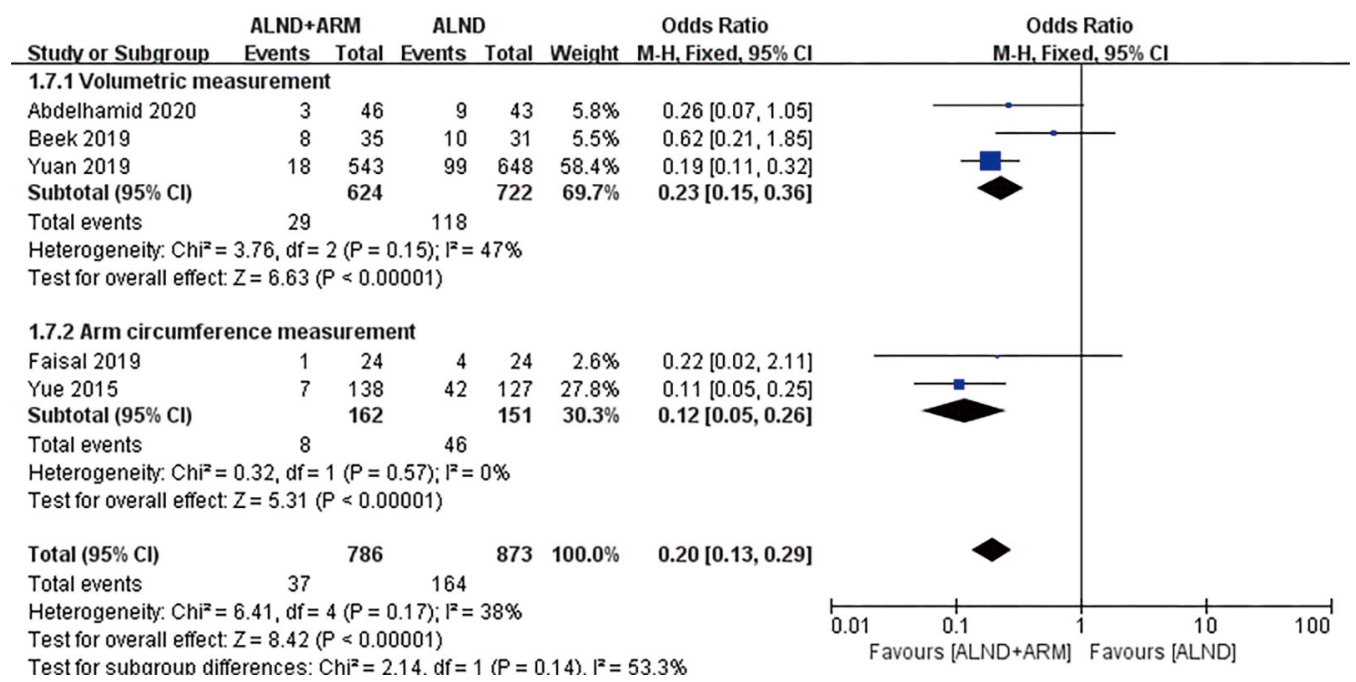

Figure 8 Forest plot for the incidence of breast cancer-related lymphedema in studies using different methods to measure arm lymphedema.

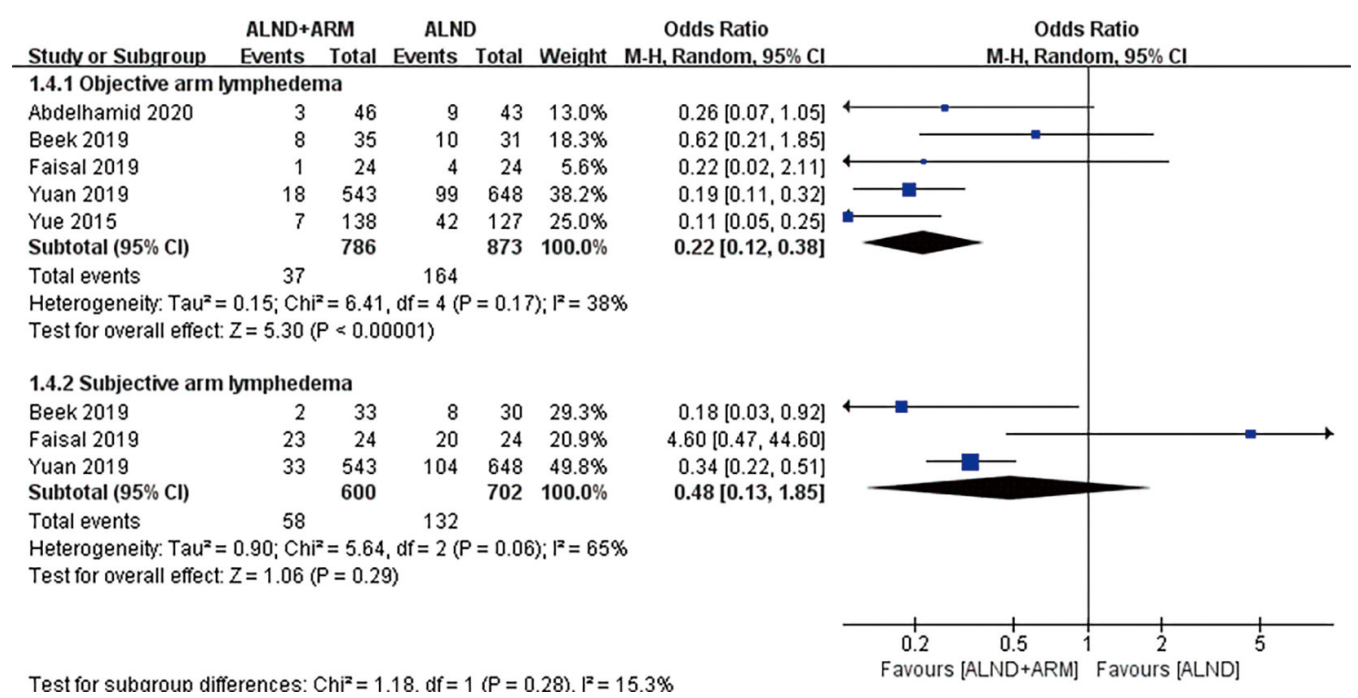

Figure 9 Forest plot for the incidence of breast cancer-related lymphedema in studies using subjective or objective measurement of arm lymphedema.

impairment of lymphatic drainage from the ipsilateral arm following surgery or radiation therapy (24). However, the pathophysiology of BCRL has not been fully illuminated and probably involves multiple factors and has a high degree of complexity. Most of BCRL are secondary to unknown or unidentified upper limb lymphatic ducts cut during the operation. According to previous studies, improving symptoms and preventing deterioration are the focus of treatment for BCRL. To date, no uniform treatment or recommendation for preventing BCRL has been established (25).

The current meta-analysis included 5 RCTs which reported the primary and secondary outcomes of BC patients who received ALND-ARM and standard-ALND alone interventions. The results showed the incidence of lymphedema to be $4.71 \%$ in the ALND plus ARM group and $18.79 \%$ in the ALND group. Previous studies have shown that the risk of upper limb lymphedema is $7 \%$ to $77 \%$ in patients receiving ALND (3). Our findings demonstrated that the incidence of BCRL with ALND 
plus ARM was significantly lower than that with ALND alone, which suggests that ALND with ARM can effectively avoid the occurrence of BCRL without impacting shoulder movement; thus, it may fundamentally solve the problem of BCRL. In most cases, the lymphatics responsible for arm drainage are positioned deep in the armpits; however, ALND often destroys deep-positioned lymphatics, which in turn induces lymphedema (26). One study investigated the location and metastasis of arm nodes, and evaluated the incidence of lymphedema following surgery to preserve the arm nodes. It showed that, with the exception of patients with a high surgical $\mathrm{N}$ stage, preservation of the arm nodes was attainable in every BC patient whose arm nodes could be identified, whether ALND or SLNB was used, and could significantly reduce the occurrence of lymphedema (27). A recent meta-analysis reviewed 29 studies, involving 4,954 patients, which clinically applied ARM in patients with BC. The results suggested that applying ARM during ALND led to a significant reduction in arm lymphedema compared to ARM resection (28). The 5 RCTs in this metaanalysis, which included 1,659 patients, were carried out with different inclusion criteria and follow-up times. The results of these studies indicate that the combination of conventional ALND with ARM to preserve nodes reduces the incidence of lymphedema.

Oncological safety is a concern in the clinical application of ARM. In our study, oncological safety was determined by the metastatic rate of ARM nodes. Two studies reported the rates of ARM node metastasis, and 1 out of 84 (1.19\%) patients who received ARM during ALND had ARM metastasis, compared with none of the participants in the ALND alone group, indicating that there was no significant difference between the 2 groups. Our results were consistent with those of previous studies $(29,30)$.

Using the limited data of the 2 included studies mentioned above, we found the positive rate of arm lymph nodes to be approximately $1.2 \%(2 / 162)$. Scientists first reported on metastasis in the upper arm lymph nodes in clinically positive patients in 2008 (16), and the results hinted that there is still a risk of lymph node metastasis with ARM. Previous studies have shown that the rate of lymph node metastasis in ARM is positively associated with the clinical stage of BC, and metastasis in the upper arm lymph nodes in patients with armpit lymph node metastasis may be involved in BC metastasis through common lymphatics (31-33). Thus, the oncological safety of preserving ARM lymph nodes as well as the corresponding lymphatics is a key issue in promoting the clinical application of ARM.
In their RCT, Yuan et al. first reported the incidence of regional recurrence between 2 groups; the rate of regional recurrence did not differ significantly between the groups during a median follow-up of 37 months (22). Moreover, a recent report reviewed the development of the ARM procedure and discussed its feasibility, safety, and relevance. It suggested that preserving the ARM lymph nodes and the corresponding lymphatics has an acceptable level of safety for patients with clinical node-negative $\mathrm{BC}$ with involved SLNs for whom complete ALND is recommended; for patients with clinical node-positive, clinicians should consider ARM-ALND for properly selected patients who are due to undergo complete axillary surgery (34). Another meta-analysis that performed a comparison of patients with stage pN0-1 and pN2-3 BC suggested that ARM should be used with caution and that for patients with stage $\mathrm{pN} 0-1 \mathrm{BC}$, there was a significant increase in the risk of metastatic ARM nodes in comparison with those with stage pN2-3 disease (35). However, because of insufficient data, the incidence of upper extremity lymphedema was not compared between the 2 groups of patients. In fact, to date, there have been no reports in the literature of an increase in axillary recurrence following preservation of ARM lymph nodes. In the present study, a pooled analysis showed that the 2 groups did not differ significantly with respect to oncological safety. Large-scale studies are needed to investigate the safety of ARM for breast cancer patients. Therefore, for advanced patients, the use of ARM during ALND should be considered carefully. Future studies should focus on selecting the $\mathrm{CPN}+$ patients who could benefit from ALND with ARM.

The subgroup analysis of follow-up period and assessment method in the 5 included RCTs also needs to be discussed. In the follow-up period subgroup analysis, the experimental and control groups did not differ significantly in terms of the incidence of BCRL during 6 months of follow-up. However, a significant difference was identified after follow up for 12 and more than 20 months. From the result of this subgroup analysis, a short duration of follow-up was likely insufficient for distinguishing transient lymphedema caused by acute surgical edema; thus, patients should be followed up for a longer period of time. Based on the results of long-term follow-up, ARM during ALND could reduce the rate of BCRL. Assessments of increased upper limb circumference and arm volume are the most important detection methods for lymphedema (36); however, patients are also accompanied by decreased upper limb activity, abnormal skin sensation, upper limb pain, 
and other lymphedema-related symptoms of discomfort, which manifest earlier than increases in circumference and volume. Therefore, it is also necessary to evaluated the lymphedema-related symptoms of discomfort, numbness, and pain. The pooled analysis which took patient subjective parameters of lymphedema-related symptoms into account showed that the incidence of BCRL did not differ significantly in relation to subjective parameters. However, we observed a lower incidence of BCRL with a significant difference favoring ARM during ALND when objective parameters were used as the main outcomes. There was obviously higher clinical heterogeneity among the 3 studies which used subjective parameters for assessment the BCRL $(2,21,22)$. This finding may be partly explained by the population differences and clinical heterogeneity, or insufficient information on preserved arm lymph nodes. The results of subjective evaluation of lymphedema can serve as reference, but stronger evidence is needed to prove the advantages of ARM in reducing BCRL.

BCRL is a common complication after comprehensive treatment of breast cancer, which seriously affects the quality of life and physical and mental health of breast cancer patients; current prevention methods for BCRL mainly include: surgical prevention, early screening, and disease Management and self-protection and exercise. Among them, arm is one of the most commonly used surgical treatments (10). The progress of arm is mainly reflected in the selection of tracers and the refinement and improvement of surgical procedures. Studies have shown that the incidence of BCRL in patients undergoing ARM axillary lymph node dissection is $5.93 \%$, which is lower than that of patients without ARM (33.07\%) (37). However, not all traced ARM lymph nodes have retention value, and some may have metastasized. For example, a fine-needle aspiration biopsy may result in false negatives $(38,39)$. Therefore, the risk of metastasis and recurrence should be considered when applying ARM, and its safety and feasibility need to be further verified. Since RCTs are considered to be the gold standard of contemporary medical research, the current meta-analysis generated conclusions about the effectiveness of ARM during ALND based on the 5 eligible RCTs. However, there were still some limitations to our study. First, there was considerable heterogeneity in study protocols, populations, assessment measures, and follow-up periods. Second, this systematic review included only a small number of studies, and a conclusion regarding the longer-term effects of ARM on the incidence of BCRL is still lacking. Due to the limited number of qualified studies, it is difficult to determine the superiority of ARM in all aspects. In future, well-designed RCTs are suggested to provide evidence on the superiority of ARM and to determine the best application of this surgical technique.

In conclusion, ARM can obviously reduce the incidence of upper limb lymphedema after surgery for BC, but there is a risk of upper limb lymph node metastasis, the rate of which is closely related to $\mathrm{BC}$ armpit lymph node stage. Therefore, in ARM for BC, the upper limb lymph nodes need to be carefully preserved. Further high-quality RCTs should be carefully designed to provide a more reliable and convincing basis for the application of ARM in BC.

\section{Acknowledgments}

Funding: Joint Construction Project of Medical Science and Technology of Henan Province (grant no. LHGJ20190635).

\section{Footnote}

Reporting Checklist: The authors have completed the PRISMA reporting checklist. Available at http://dx.doi. org/10.21037/gs-21-186

Conflicts of Interest: Both authors have completed the ICMJE uniform disclosure form (available at http://dx.doi. org/10.21037/gs-21-186). The authors have no conflicts of interest to declare.

Ethical Statement: The authors are accountable for all aspects of the work in ensuring that questions related to the accuracy or integrity of any part of the work are appropriately investigated and resolved.

Open Access Statement: This is an Open Access article distributed in accordance with the Creative Commons Attribution-NonCommercial-NoDerivs 4.0 International License (CC BY-NC-ND 4.0), which permits the noncommercial replication and distribution of the article with the strict proviso that no changes or edits are made and the original work is properly cited (including links to both the formal publication through the relevant DOI and the license). See: https://creativecommons.org/licenses/by-nc-nd/4.0/.

\section{References}

1. Siegel RL, Miller KD, Jemal A. Cancer statistics, 2020. CA Cancer J Clin 2020;70:7-30. 
2. Beek MA, Gobardhan PD, Klompenhouwer EG, et al. A patient- and assessor-blinded randomized controlled trial of axillary reverse mapping (ARM) in patients with early breast cancer. Eur J Surg Oncol 2020;46:59-64.

3. Ahmed M, Rubio IT, Kovacs T, et al. Systematic review of axillary reverse mapping in breast cancer. Br J Surg 2016;103:170-8.

4. Lucci A, McCall LM, Beitsch PD, et al. Surgical complications associated with sentinel lymph node dissection (SLND) plus axillary lymph node dissection compared with SLND alone in the American College of Surgeons Oncology Group Trial Z0011. J Clin Oncol 2007;25:3657-63.

5. Klimberg VS. A new concept toward the prevention of lymphedema: axillary reverse mapping. J Surg Oncol 2008;97:563-4.

6. Thompson M, Korourian S, Henry-Tillman R, et al. Axillary reverse mapping (ARM): a new concept to identify and enhance lymphatic preservation. Ann Surg Oncol 2007;14:1890-5.

7. Tummel E, Ochoa D, Korourian S, et al. Does Axillary Reverse Mapping Prevent Lymphedema After Lymphadenectomy? Ann Surg 2017;265:987-92.

8. Schunemann E, Jr., Doria MT, Silvestre JB, et al. Prospective study evaluating oncological safety of axillary reverse mapping. Ann Surg Oncol 2014;21:2197-202.

9. Tausch C, Baege A, Dietrich D, et al. Can axillary reverse mapping avoid lymphedema in node positive breast cancer patients? Eur J Surg Oncol 2013;39:880-6.

10. Khan SA. Axillary reverse mapping to prevent lymphedema after breast cancer surgery: defining the limits of the concept. J Clin Oncol 2009;27:5494-6.

11. Shao X, Sun B, Shen Y. Axillary reverse mapping (ARM): where to go. Breast Cancer 2019;26:1-10.

12. Abdelhamid MI, Bari AA, Farid MI, et al. Evaluation of axillary reverse mapping (ARM) in clinically axillary node negative breast cancer patients - Randomised controlled trial. Int J Surg 2020;75:174-8.

13. Deng H, Chen L, Jia W, et al. Safety study of axillary reverse mapping in the surgical treatment for breast cancer patients. J Cancer Res Clin Oncol 2011;137:1869-74.

14. Noguchi M, Noguchi M, Nakano Y, et al. Axillary reverse mapping using a fluorescence imaging system in breast cancer. J Surg Oncol 2012;105:229-34.

15. Connor C, McGinness M, Mammen J, et al. Axillary reverse mapping: a prospective study in women with clinically node negative and node positive breast cancer. Ann Surg Oncol 2013;20:3303-7.
16. Nos C, Kaufmann G, Clough KB, et al. Combined axillary reverse mapping (ARM) technique for breast cancer patients requiring axillary dissection. Ann Surg Oncol 2008; 15:2550-5.

17. Moher D, Liberati A, Tetzlaff J, et al. Preferred reporting items for systematic reviews and meta-analyses: the PRISMA statement. PLoS Med 2009;6:e1000097.

18. Higgins JP, Altman DG, Gotzsche PC, et al. The Cochrane Collaboration's tool for assessing risk of bias in randomised trials. BMJ 2011;343:d5928.

19. Higgins JP, Thompson SG, Deeks JJ, et al. Measuring inconsistency in meta-analyses. BMJ 2003;327:557-60.

20. Ioannidis JP. Interpretation of tests of heterogeneity and bias in meta-analysis. J Eval Clin Pract 2008;14:951-7.

21. Faisal M, Sayed MG, Antonious K, et al. Prevention of lymphedema via axillary reverse mapping for arm lymphnode preservation following breast cancer surgery: a randomized controlled trial. Patient Saf Surg 2019;13:35.

22. Yuan Q, Wu G, Xiao SY, et al. Identification and Preservation of Arm Lymphatic System in Axillary Dissection for Breast Cancer to Reduce Arm Lymphedema Events: A Randomized Clinical Trial. Ann Surg Oncol 2019;26:3446-54.

23. Yue T, Zhuang D, Zhou P, et al. A Prospective Study to Assess the Feasibility of Axillary Reverse Mapping and Evaluate Its Effect on Preventing Lymphedema in Breast Cancer Patients. Clin Breast Cancer 2015;15:301-6.

24. Purushotham AD, Bennett Britton TM, Klevesath $\mathrm{MB}$, et al. Lymph node status and breast cancer-related lymphedema. Ann Surg 2007;246:42-5.

25. Li L, Yuan L, Chen X, et al. Current Treatments for Breast Cancer-Related Lymphoedema: A Systematic Review. Asian Pac J Cancer Prev 2016;17:4875-83.

26. Seyednejad N, Kuusk U, Wiseman SM. Axillary reverse lymphatic mapping in breast cancer surgery: a comprehensive review. Expert Rev Anticancer Ther 2014;14:771-81.

27. Han JW, Seo YJ, Choi JE, et al. The efficacy of arm node preserving surgery using axillary reverse mapping for preventing lymphedema in patients with breast cancer. $\mathrm{J}$ Breast Cancer 2012;15:91-7.

28. Wijaya WA, Peng J, He Y, et al. Clinical application of axillary reverse mapping in patients with breast cancer: A systematic review and meta-analysis. Breast 2020;53:189-200.

29. Boneti C, Korourian S, Bland K, et al. Axillary reverse mapping: mapping and preserving arm lymphatics may be important in preventing lymphedema during sentinel 
lymph node biopsy. J Am Coll Surg 2008;206:1038-42; discussion 1042-4.

30. Ponzone R, Mininanni P, Cassina E, et al. Axillary reverse mapping in breast cancer: can we spare what we find? Ann Surg Oncol 2008;15:390-1; author reply 392-3.

31. Noguchi M. Axillary reverse mapping for breast cancer. Breast Cancer Res Treat 2010;119:529-35.

32. Khandelwal R, Poovamma CU, Shilpy C, et al. Axillary reverse mapping: Is it feasible in locally advanced breast cancer patients? Breast Dis 2014;34:151-5.

33. Ponzone R, Cont NT, Maggiorotto F, et al. Extensive nodal disease may impair axillary reverse mapping in patients with breast cancer. J Clin Oncol 2009;27:5547-51.

34. Beek MA, Gobardhan PD, Schoenmaeckers EJ, et al. Axillary reverse mapping in axillary surgery for breast cancer: an update of the current status. Breast Cancer Res Treat 2016;158:421-32.

35. Han C, Yang B, Zuo WS, et al. The Feasibility and

Cite this article as: Guo X, Jiao D, Zhu J, Xiao H, Zhao X, Yang Y, Zhao Y, Liu Z. The effectiveness of axillary reverse mapping in preventing breast cancer-related lymphedema: a meta-analysis based on randomized controlled trials. Gland Surg 2021;10(4):1447-1459. doi: 10.21037/gs-21-186
Oncological Safety of Axillary Reverse Mapping in Patients with Breast Cancer: A Systematic Review and Meta-Analysis of Prospective Studies. PLoS One 2016;11:e0150285.

36. DiSipio T, Rye S, Newman B, et al. Incidence of unilateral arm lymphoedema after breast cancer: a systematic review and meta-analysis. Lancet Oncol 2013;14:500-15.

37. Yue T, Zhuang DY, Zhou P, et al. A prospective study to assess the feasibility of axillary reverse mapping and evaluate its effect on preventing lymphedema in breast cancer patients. Clin Breast Cancer 2015;15:301-6.

38. Han C, Yang B, Zuo WS, et al. The feasibility and oncological safety of axillary reverse mapping in patients with breast cancer: A systematic review and meta-analysis of prospective studies. PLoS One 2016;11:e0150285.

39. Tummel E, Ochoa D, Korourian S, et al. Does axillary reverse mapping prevent lymphedema after lymphadenectomy? Ann Surg 2017;265:987-92 . 


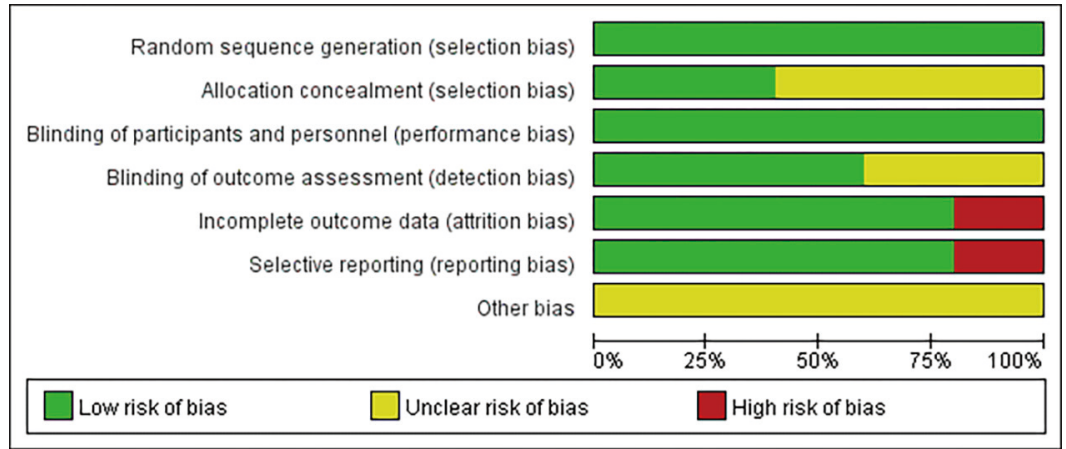

Figure S1 Risk of bias summary: The risk of bias tool incorporates the assessment of randomization (sequence generation and allocation concealment), blinding (participants and outcome assessors), incomplete outcome data, selective outcome reporting, and other risk of bias. The items were classified as "low risk" "unclear risk" or "high risk", with red, green, and yellow representing "high risk", "low risk", and "unclear risk", respectively. 